\title{
ON ALGEBRAS WITH A FINITE NUMBER OF INDECOMPOSABLE MODULES
}

\author{
BY \\ C. W. $\operatorname{CURTIS}\left({ }^{1}\right)$ AND J. P. JANS $\left({ }^{2}\right)$
}

Let $A$ be an associative algebra over a field $K$ such that the dimension, $[A: K]$, of $A$ over $K$ is finite. Following the terminology of [8], we shall say that $A$ is of bounded module type if there exists an integer $n$ such that for every indecomposable left $A$-module $M$, the inequality $[M: K] \leqq n$ holds. The algebra $A$ is of finite module type if $A$ has only a finite number of nonisomorphic indecomposable left modules. Clearly, algebras of finite module type are of bounded module type. The converse to this statement has been conjectured for some time [8], and it appears to be quite difficult to prove. There are some classes of algebras (more general than semi-simple algebras) which are known to be of finite module type. Higman has shown [7] that the group algebra $K G$ of a finite group $G$ over a field $K$ of characteristic $p$ is of finite module type if and only if the $p$-Sylow groups of $G$ are cyclic. Nakayama [13] introduced the class of generalized uniserial algebras, and showed that they are all of finite module type. It has been known for some time, however, that these two classes do not contain all algebras of finite module type [16].

Before stating the main theorem of this paper, we introduce some notations and definitions. We shall be concerned only with finite dimensional algebras. We shall denote the radical of an algebra $A$ by $N$. By the socle $S(M)$ of a left $A$-module $M$, we mean the sum of the simple submodules of $M ; S(M)$ can also be characterized as the set of all elements $m$ in $M$ such that $N m=0$. The socle $S(M)$ of $M$ is the maximal semi-simple submodule of $M$, and is a direct sum of simple modules. In general, a semisimple module will be called square-free if it is a direct sum of simple modules, no two of which are isomorphic. Note that in a square-free semisimple module $M$, two simple submodules are isomorphic if and only if they coincide.

We can now state the main theorem of this paper.

Received by the editors July 3, 1963.

$\left.{ }^{1}\right)$ The research of Curtis was supported by the National Science Foundation, grant GP-280. This paper supersedes the paper [3] presented to the American Mathematical Society by Curtis on January 25, 1963.

$\left({ }^{2}\right)$ The research of Jans was supported by the National Science Foundation, grant G19915. 
Theorem. Let $A$ be an algebra over a field $K$ such that the following conditions are satisfied:

(a) $K$ is algebraically closed.

(b) The socle of every indecomposable left A-module is square-free.

Then $A$ is of finite module type.

We shall show first that the hypothesis (b) implies that $A$ is of bounded module type. Then we shall prove a series of lemmas which will lead to a proof of the theorem. At the end of the paper we shall give a corollary to the theorem, and some other remarks and examples.

Lemma A. Let $A$ be an algebra over a field $K$ such that for every indecomposable left $A$-module $M, S(M)$ is square-free. Then $A$ is of bounded module type.

Proof. Let $T$ be the direct sum of a set of representatives of the distinct isomorphism classes of simple left $A$-modules, and let $H(T)$ be the injective hull [5] of $T$. Then $[H(T): K]$ is finite, and we shall prove that every indecomposable left $A$-module is isomorphic to a submodule of $H(T)$. Because of the definition of $T$ and the hypothesis of the lemma, if $M$ is indecomposable, there is a monomorphism $\alpha: S(M) \rightarrow T$, and hence we have a diagram with an exact row

$$
\begin{gathered}
0 \rightarrow S(M) \rightarrow M \\
\alpha \downarrow \\
H(T)
\end{gathered}
$$

which can be completed to a commutative diagram

$$
\begin{aligned}
0 \rightarrow & S(M) \rightarrow M \\
& \alpha \downarrow \\
& H(T)
\end{aligned}
$$

since $H(T)$ is injective. Then $\mu$ is a monomorphism, for the following reason. Let $U$ be a submodule of $M$ such that $\mu(U)=0$. If $U \neq 0$, then $U$ contains a submodule $U_{1} \neq 0$ such that $N U_{1}=0$, and $U_{1} \subset S(M)$, contrary to the assumption that the restriction of $\mu$ to $S(M)$ is a monomorphism. This completes the proof of the lemma.

A module $M$ with a composition series is said to have finite length; the number of composition factors is called the length of $M$, and is denoted by $c(M)$. If $P$ and $Q$ are submodules of $M$, then the isomorphism theorem $(P+Q) / Q \cong P /(P \cap Q)$ implies that

$$
c(P)+c(Q)=c(P+Q)+c(P \cap Q) .
$$

This formula is useful for detecting direct sums. For if $P+Q=M$, and $c(P)+c(Q) \leqq c(M)$, then it is clear that $c(P \cap Q)=0$, and $M=P \oplus Q$. We shall also need the fact, which depends only on the modular law in 
the lattice of submodules, and not on a finiteness assumption, that if $B=C \oplus D$, and $W$ is a submodule of $C$, then $B / W \cong(C / W) \oplus D$.

Lemma B. Let $A$ be an algebra such that for each indecomposable left $A$ module $M, S(M)$ is square-free. Let $M$ be a fixed indecomposable module, and $C$ a simple module. Suppose that for some indecomposable module $E$ there is an exact sequence

$$
0 \rightarrow C \stackrel{\mu}{\rightarrow} E \stackrel{\pi}{\rightarrow} M \rightarrow 0 .
$$

Then for any other module $E^{\prime}$ which can be fitted into an exact sequence

$$
0 \rightarrow C \stackrel{\mu^{\prime}}{\rightarrow} E^{\prime} \stackrel{\pi^{\prime}}{\rightarrow} M \rightarrow 0,
$$

either $E^{\prime} \cong E$, or the sequence splits.

Proof. Let $P$ be the projective cover (see [6]) of $M$. Then there is an epimorphism $\rho: P \rightarrow M$ whose kernel $L$ is contained in $N P$, where $N$ is the radical of $A$. Letting $\lambda: L \rightarrow P$ be the injection map, we have an exact sequence

$$
0 \rightarrow L \stackrel{\lambda}{\rightarrow} P \stackrel{\rho}{\rightarrow} M \rightarrow 0 .
$$

Moreover, $M$ has finite length because of Lemma A, and hence $P$ has finite length.

We observe next that there exist commutative diagrams

$$
\begin{aligned}
& 0 \rightarrow L \stackrel{\lambda}{\rightarrow} P \stackrel{\rho}{\rightarrow} M \rightarrow 0
\end{aligned}
$$

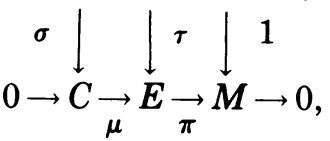

and

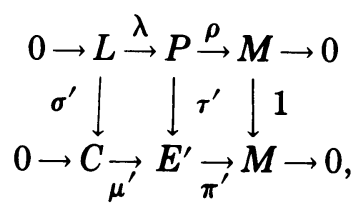

where $\tau$ and $\tau^{\prime}$ are filled in using the fact that $P$ is projective, and $\sigma$ exists, for example, because $\pi \tau \lambda(L)=0$, so that $\tau \lambda(L) \subset \operatorname{Ker} \pi=\operatorname{Im} \mu$, by the exactness of the lower sequence.

We can assume that neither lower sequence splits, otherwise there is nothing to prove.

We prove first that $\tau$ and $\tau^{\prime}$ are epimorphisms. In the case of $\tau$, we begin with the observation that $E=\operatorname{Im} \tau+\operatorname{Ker} \pi$, since $e \in E$ implies that 
$\pi(e)=\rho(p)$ for some $p \in P$. Then $\pi \tau(p)=\pi(e), e-\tau(p) \in \operatorname{Ker} \pi$, and $e \in \operatorname{Im} \tau+\operatorname{Ker} \pi$. Since $\operatorname{Ker} \pi=\operatorname{Im} \mu \cong C$ is simple, $\operatorname{Im} \tau \cap \operatorname{Ker} \pi$ is either Ker $\pi$ or zero. If it is zero, then $E=\operatorname{Im} \tau \oplus \operatorname{Ker} \pi$, contrary to the assumption that the sequence $0 \rightarrow C \rightarrow E \rightarrow M \rightarrow 0$ does not split. Therefore Ker $\pi$ $C \operatorname{Im} \tau$, and $E=\operatorname{Im} \tau$. Similarly $E^{\prime}=\operatorname{Im} \tau^{\prime}$.

Let $F=\operatorname{Ker} \tau, H=\operatorname{Ker} \tau^{\prime}$. If $F=H$, then $E \cong P / F \cong P / H \cong E^{\prime}$, and the lemma is proved. In case $F \neq H$, we shall prove that the indecomposability of $M=P / L$, and the hypothesis that indecomposable modules have square-free socles, imply that $E=P / F$ has a nontrivial direct decomposition, so that the case $F \neq H$ cannot occur.

The problem can be formulated entirely in terms of the submodules $N P, L, F=\operatorname{Ker} \tau, H=\operatorname{Ker} \tau^{\prime}$, and $X=F \cap H$ of $P$. We have $L \subset N P$ because $P$ is the projective cover of $M$. Since

$$
F=\operatorname{Ker} \tau \subset \operatorname{Ker} \pi \tau=\operatorname{Ker} \rho=\operatorname{Im} \lambda,
$$

we have $F \subset L$, and similarly $H \subset L$. Finally we have isomorphisms $L / F \cong C, L / H \cong C$, and $L / X \cong C \oplus C$. Most of this information is summarized in the diagram

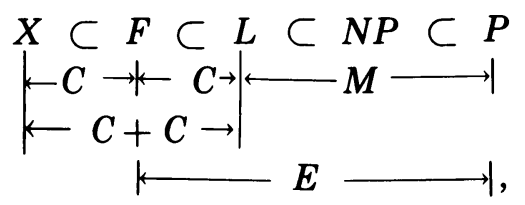

in which the expressions $|\leftarrow \rightarrow|$ indicate factor modules. Our objective is to show that $E=P / F$ decomposes. There is no loss of generality in assuming that $X=0$.

Since $S(P) \supset L \cong C \oplus C, P$ is not indecomposable. Therefore there exist submodules $X_{1}, \cdots, X_{r}$, for some $r \geqq 2$, such that $P=X_{1} \oplus \cdots \oplus X_{r}$, and each $X_{i}$ is indecomposable and different from zero.

For each $i$, let $X_{i}^{\prime}$ be the submodule $\sum_{j \neq i} X_{j}$; then we have $P=X_{i} \oplus X_{i}^{\prime}$, and $c(P)=c\left(X_{i}\right)+c\left(X_{i}^{\prime}\right)$, for $i=1, \cdots, r$.

The next step will be to show that $X_{i}^{\prime} \cap L$ is strictly between 0 and $L$, for $i=1, \cdots, r$.

First, if $X_{i}^{\prime} \cap L=0$, then $\left(X_{i}^{\prime}+L\right) / X_{i}^{\prime} \cong L \cong C \oplus C$, and the module $P / X_{i}^{\prime} \cong X_{i}$ has a socle containing two copies of $C$, contrary to the hypothesis that socles of indecomposable modules are square-free.

Now suppose that $X_{i}^{\prime} \cap L=L$. Then $L \subset X_{i}^{\prime}$, and by the remark preceding the lemma, we have a direct sum decomposition

$$
P / L \cong X_{i} \oplus\left(X_{i}^{\prime} / L\right) \text {. }
$$


Since $P / L=M$ is indecomposable and $X_{i} \neq 0$, we have $X_{i}^{\prime}=L$. Then $c\left(X_{i}^{\prime}\right)=2$, and $c\left(\left(X_{i}+F\right) / F\right) \leqq c\left(X_{i}\right)=c(P)-c\left(X_{i}^{\prime}\right)=c(P)-2$. Since $P / F=\left(X_{i}+F\right) / F+L / F$, and $L / F$ is simple, it follows that $P / F$ $=\left(X_{i}+F\right) / F \oplus L / F$, otherwise $c(P / F)=c\left(\left(X_{i}+F\right) / F\right) \leqq c(P)-2$, which is impossible because $c(P / F)=c(P)-1$. Thus the exact sequence

$$
0 \rightarrow L / F \rightarrow P / F \rightarrow P / L \rightarrow 0
$$

splits, contrary to the assumption that the original sequence $0 \rightarrow C \rightarrow E$ $\rightarrow M \rightarrow 0$ does not split. This completes the proof that $X_{i}^{\prime} \cap L$ is strictly between 0 and $L$, for $i=1, \cdots, r$.

Now we shall show that for each $i, X_{i}$ contains a copy of $C$. Since $X_{i}^{\prime} \cap L \neq L$, it follows that

$$
\left(X_{i}^{\prime}+L\right) / X_{i}^{\prime} \cong L /\left(X_{i}^{\prime} \cap L\right) \neq 0 .
$$

But $L \cong C \oplus C$, and hence $\left(X_{i}^{\prime}+L\right) / X_{i}^{\prime} \cong C$. Then $X_{i} \cong P / X_{i}^{\prime}$ contains $\left(X_{i}^{\prime}+L\right) / X_{i}^{\prime}$, and we have shown that $X_{i}$ contains a copy of $C$.

We distinguish now the two cases in which the number $r$ of summands $X_{i}$ is two or is greater than two. First suppose $r=2$. Then $X_{1}^{\prime}=X_{2}$ and $X_{2}^{\prime}=X_{1}$. If one of the modules $X_{i}$ contains $F$, say $X_{1}$, then by the modular law we have a direct decomposition

$$
P / F \cong\left(X_{1} / F\right) \oplus X_{2} .
$$

Note that this is a nontrivial decomposition, for if $X_{1}=F$, then $P / X_{2} \cong F$ $\cong C$ is simple, and hence $X_{2} \supset N P \supset L \supset F$, and $X_{1} \cap X_{2} \supset F$, contrary to the fact that $X_{1} \cap X_{2}=0$. Thus we have a decomposition of $E \cong P / F$ in this case.

If neither $X_{1}$ nor $X_{2}$ contains $F$, then for each $i, X_{i} \cap L \neq F$. Since we have shown that $X_{i}^{\prime} \cap L \neq 0$, and $c(L)=2$, it follows that $\left(X_{i} \cap L\right)+F$ $=L$. Therefore $\left(X_{1}+F\right) \cap\left(X_{2}+F\right) \supset L$. On the other hand, the formula preceding the lemma implies that $c\left(\left(X_{1}+F\right) \cap\left(X_{2}+F\right)\right)=2$, and because $c(L)=2$, we have $\left(X_{1}+F\right) \cap\left(X_{2}+F\right)=L$. Therefore we have a directsum decomposition

$$
P / L \cong\left(X_{1}+F\right) / L \oplus\left(X_{2}+F\right) / L .
$$

This will contradict the indecomposability of $M \cong P / L$ if we can prove it is a nontrivial decomposition. But $X_{1}+F=L$ implies $X_{1} \subset L$, and since $X_{1}=X_{2}^{\prime}, \quad X_{1} \neq L$. Then $P / X_{2} \cong X_{1}$ is simple, and $X_{2} \supset N P \supset L \supset F$, contrary to assumption. Thus the case $r=2$, with neither $X_{1}$ nor $X_{2}$ containing $F$, cannot occur.

Finally we have the case $r \geqq 3$. Since each $X_{i}$ contains a copy of $C$, it follows that $S(P)$ contains the direct sum of at least $r$ copies of $C$. But since $P / F$ is $P$ with one copy of $C$ factored out, it follows that $S(P / F)$ 
contains the direct sum of at least $r-1 \geqq 2$ copies of $C$, and by the hypothesis of the lemma, $E \cong P / F$ is not indecomposable. This completes the proof of the lemma.

REMARK. The preceding lemma was suggested by the following connection between the lemma and the functor $\operatorname{Ext}_{A}^{1}(M, C)$ (see [2] or [9]). The functor property of $\operatorname{Ext}_{A}^{1}(M, C)$ in the second variable implies that $\operatorname{Ext}_{A}^{1}(M, C)$ is a module over the endomorphism ring $E(C)$ of $C$. If $C$ is a simple module, then $E(C)$ is a division ring, and $\operatorname{Ext}_{A}^{1}(M, C)$ is a vector space over $E(C)$. If the dimension of this space is not greater than one, we shall prove first that there are at most two nonisomorphic modules $E$ which can be fitted into an exact sequence $0 \rightarrow C \rightarrow E \rightarrow M \rightarrow 0$. It is sufficient, in case the dimension is one, to show that for two nonsplit exact sequences

$$
0 \rightarrow C \stackrel{\phi}{\rightarrow} E \stackrel{\psi}{\rightarrow} M \rightarrow 0
$$

and

$$
0 \rightarrow C \stackrel{\phi^{\prime}}{\rightarrow} E^{\prime} \stackrel{\psi^{\prime}}{\rightarrow} M \rightarrow 0
$$

we have $E \cong E^{\prime}$. These sequences correspond to nonzero elements $e$ and $e^{\prime}$ in $\operatorname{Ext}_{A}^{1}(M, C)$, and hence there exists an automorphism $\mu$ of $C$ which carries $e$ to $e^{\prime}$. This is equivalent (see $[14, \S 1]$ ) to the existence of a commutative diagram

$$
\begin{aligned}
& 0 \rightarrow C \stackrel{\phi}{\rightarrow} E \stackrel{\psi}{\rightarrow} M \rightarrow 0 \\
& \mu|\theta| \downarrow 1 \\
& 0 \rightarrow C \underset{\phi^{\prime}}{\underset{\psi^{\prime}}{\rightarrow}} M \rightarrow 0 \text {, }
\end{aligned}
$$

and by the five-lemma [2, p. 5] $\theta$ is an isomorphism.

The proof of Lemma B establishes the following result. Suppose the socles of all indecomposable $A$-modules are square-free, and let $0 \rightarrow C \rightarrow E$ $\rightarrow M \rightarrow 0$ be an exact sequence, with $E$ and $M$ indecomposable, and $C$ simple. Then the dimension of $\operatorname{Ext}_{A}^{1}(M, C)$ over $E(C)$ is $\leqq 1$. Suppose, to the contrary, that the dimension is $\geqq 2$. Form the exact sequence

$$
0 \rightarrow L \rightarrow P \rightarrow M \rightarrow 0
$$

as in Lemma B. Since $P$ is projective, there is an epimorphism $\operatorname{Hom}(L, C)$ $\rightarrow \operatorname{Ext}_{A}^{1}(M, C)$, so that the dimension of $\operatorname{Hom}(L, C)$ over $E(C)$ is $\geqq 2$. Form the commutative diagram (see Lemma B)

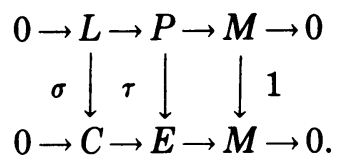


Because the dimension of $\operatorname{Hom}(L, C)$ over $E(C)$ is $\geqq 2$, there exists $\sigma^{\prime} \neq 0$ in $\operatorname{Hom}(L, C)$ such that $\operatorname{Ker} \sigma \neq \operatorname{Ker} \sigma^{\prime}$. The proof of Lemma B then shows that $E$ decomposes, contrary to hypothesis. Therefore the dimension of $\operatorname{Ext}_{A}^{1}(M, C)$ over $E(C)$ is not greater than 1 .

In the proof of the next lemma, we use for the first time the hypothesis that the field $K$ is algebraically closed.

Lemma C. Let $A$ be an algebra over an algebraically closed field $K$ such that the socle of every indecomposable left A-module is square-free. Let

$$
0 \rightarrow C \rightarrow E \rightarrow M \rightarrow 0
$$

be an exact sequence of $A$-modules, such that $C$ is simple, $E$ is indecomposable, and $M$ is not indecomposable. Then there exist modules $X_{1}$ and $X_{2}$ with $c\left(X_{i}\right)$ $<c(E), i=1,2$, and an exact sequence

$$
0 \rightarrow C \stackrel{\eta}{\rightarrow} X_{1} \oplus X_{2} \rightarrow E \rightarrow 0
$$

such that if

$$
0 \rightarrow C \stackrel{\eta^{\prime}}{\rightarrow} X_{1} \oplus X_{2} \rightarrow E^{\prime} \rightarrow 0
$$

is another exact sequence with $E^{\prime}$ indecomposable, then $E \cong E^{\prime}$.

Proof. We may assume $C \subset E$, and $M=E / C$. Since $M$ is decomposable, there exist submodules $X_{i} \supset C, i=1,2$ such that $E=X_{1}+X_{2}$, and $X_{1}$ $\cap X_{2}=C$. Since $S(E)$ is square-free, each $X_{i}$ contains a unique submodule $C_{i}$ isomorphic to $C$. We have an exact sequence

$$
0 \rightarrow C \stackrel{\eta}{\rightarrow} X_{1} \oplus X_{2} \stackrel{\lambda}{\rightarrow} E \rightarrow 0,
$$

since $X_{1} \cap X_{2}=C$. Now suppose we have an exact sequence

$$
0 \rightarrow C \stackrel{\eta^{\prime}}{\rightarrow} X_{1} \oplus X_{2} \stackrel{\lambda^{\prime}}{\rightarrow} E^{\prime} \rightarrow 0,
$$

with $E^{\prime}$ indecomposable. Letting $\pi_{1}$ and $\pi_{2}$ be the projections of $X_{1} \oplus X_{2}$ upon $X_{1}$ and $X_{2}$, the indecomposability of $E^{\prime}$ together with the modular law (see the remark preceding Lemma B) imply that $\pi_{i} \eta^{\prime}(C) \neq 0, i=1,2$, so that $\pi_{i} \eta^{\prime}(C)=C_{i}, i=1,2$. Then for $c \in C$, the map $\pi_{1} \eta^{\prime}(c) \rightarrow \pi_{2} \eta^{\prime}(c)$ is an isomorphism $\theta^{\prime}$ of $C_{1}$ onto $C_{2}$, and $T^{\prime}=\operatorname{Ker} \lambda^{\prime}=\left\{\eta^{\prime}(c): c \in C\right\}$ $=\left\{\pi_{1} \eta^{\prime}(c)+\pi_{2} \eta^{\prime}(c): c \in C\right\}=\left\{x+\theta^{\prime}(x): x \in C_{1}\right\}$. If $\theta^{\prime \prime}$ is another isomorphism of $C_{1}$ onto $C_{2}$, and $T^{\prime \prime}=\left\{x+\theta^{\prime \prime}(x): x \in C_{1}\right\}$, then it is sufficient to prove that $\left(X_{1}+X_{2}\right) / T^{\prime} \cong\left(X_{1}+X_{2}\right) / T^{\prime \prime}$. Because $K$ is algebraically closed and $C_{1}$ is simple, there is a fixed element $\xi \in K$ such that $\theta^{\prime \prime}(x)$ $=\xi \theta^{\prime}(x), x \in C_{1}$. Let $\xi_{L}$ denote the map $x_{2} \rightarrow \xi x_{2}$ of $X_{2}$ onto $X_{2}$, and set $h=1 \oplus \xi_{L}$. Then $h$ is an $A$-automorphism of $X_{1} \oplus X_{2}$ such that $h\left(T^{\prime}\right)=T^{\prime \prime}$. 
The isomorphism of the factor modules by $T^{\prime}$ and $T^{\prime \prime}$ follows, and the lemma is proved.

Now we can proceed with the proof of the main theorem. We prove first that for each integer $n \geqq 0$, there are at most finitely many nonisomorphic $A$-modules of length $\leqq n$. We use induction on the length. There are at most finitely many nonisomorphic modules of length one; in fact any ring with minimum condition has this property.

Suppose now that for some integer $k \leqq n$, there are only finitely many nonisomorphic modules of length $<k$, and let $E$ be a module of length $k$. We show that there are only finitely many possibilities for $E$. If $E$ is decomposable, then the induction hypothesis implies that there are only a finite number of possible isomorphism classes for $E$.

So we may assume $E$ is indecomposable, and not simple. Let $C$ be a simple submodule of $E$, and form the exact sequence

$$
0 \rightarrow C \rightarrow E \rightarrow E / C \rightarrow 0 \text {. }
$$

Since $c(E / C)<k$, there are only finitely many possibilities for $E / C$.

Case (i). If $E / C$ is indecomposable, then by Lemma $\mathrm{B}, E$ is uniquely determined by $C$ and $E / C$.

Case (ii). If $E / C$ is not indecomposable, then by Lemma $\mathrm{C}, E$ is uniquely determined by $C$ and modules of length $<k$.

This completes the proof that for each $n, A$ has only a finite number of nonisomorphic modules of length $\leqq n$. By Lemma $\mathrm{A}, A$ is of bounded module type, and it follows that $A$ is of finite module type. This completes the proof of the theorem.

Up to now we have treated only left modules. We obtain an interesting corollary to our theorem by exploiting the duality between left and right modules over a finite dimensional algebra $A$ over a field $K$. More specifically, we have a functor ( $)^{*}: M \rightarrow M^{*}$ between the category of finitely generated left $A$-modules and the category of finitely generated right $A$ modules, where $M^{*}=\operatorname{Hom}_{K}(M, K)$. The functor ( $)^{*}$ has the following properties:

(1) ()$^{*}$ is an exact contravariant functor.

(2) $\left(()^{*}\right)^{*}$ is naturally equivalent to the identity functor.

(3) $M^{*}$ is indecomposable if and only if $M$ is indecomposable.

(4) $M^{*}$ is simple if and only if $M$ is simple.

(5) For a left module $M,(M / N M)^{*} \cong S\left(M^{*}\right),(S(M))^{*} \cong M^{*} / M^{*} N$.

(5') For a right module $M,(M / M N)^{*} \cong S\left(M^{*}\right),(S(M))^{*} \cong M^{*} / N M^{*}$.

The proofs of (1) and (2) are standard arguments with dual vector spaces. (3) and (4) follow from (1) and (2). (5) and (5') can be deduced from the facts that $X$ is maximal in $M$ if and only if $(M / X)^{*}$ is simple, and that $N M$ is the intersection of the maximal submodules in $M$. 
From the above facts, particularly (3), it is clear that an algebra has as many indecomposable left modules as indecomposable right modules. Statements (5) and $\left(5^{\prime}\right)$ above allow us to state the following corollary to our main theorem.

Corollary. Let $A$ be an algebra over a field $K$ such that

(a) $K$ is algebraically closed;

(b) for every indecomposable left module $M, M / N M$ is square-free.

Then $A$ is of finite module type.

Proof. By (5) and (4) above, $M / N M$ is square-free if and only if $S\left(M^{*}\right)$ is square-free. But then the main theorem applied to right modules shows that $A$ has only a finite number of nonisomorphic indecomposable right modules. By duality, $A$ has only a finite number of nonisomorphic indecomposable left modules.

We conclude with some miscellaneous remarks and examples.

(1) The preliminary results, Lemmas $A$ and $B$, hold for rings with minimum condition, as well as for algebras. The only place in our proof where we used the fact that we had an algebra over an algebraically closed field was in Lemma $\mathrm{C}$, where we had to extend an automorphism of a simple submodule of a module with square-free socle to an automorphism of the module itself. Our main theorem holds for rings with minimum condition if we replace the hypothesis about algebraic closure by the hypothesis that the above kind of automorphisms can be extended.

(2) It is a known result [7], [4] that group algebras of bounded module type have the property that every indecomposable left module is isomorphic to a left ideal in the algebra. It is an open question whether group algebras of bounded module type satisfy the hypothesis of our main theorem. More generally, let $A$ be a finite dimensional algebra with the property that every indecomposable left module is isomorphic to a left ideal in $A$. Does $A$ satisfy the condition that the socles of indecomposable modules are squarefree? We do not answer this question, but will show that such an algebra is always a quasi-Frobenius algebra (see Chapter VIII of [4]). Let $M$ be a simple left $A$-module, and let $H(M)$ be the injective hull of $M$. Then $H(M)$ is indecomposable, injective, and by the hypothesis, is isomorphic to a left ideal $\hat{M}$ in $A$. Since $\hat{M}$ is injective, $\hat{M}$ is an indecomposable direct summand of the left regular module ${ }_{A} A$, and hence $\hat{M}$ is a principal indecomposable $A$-module. The uniqueness of the injective hull implies that if $M_{1}$ and $M_{2}$ are simple left $A$-modules, then $\hat{M}_{1} \cong \hat{M}_{2}$ if and only if $M_{1} \cong M_{2}$. Thus there are as many nonisomorphic, injective, principal indecomposable $A$ modules as there are nonisomorphic simple $A$-modules. It follows that all principal indecomposable $A$-modules are injective, and hence $A$ is a quasi- 
Frobenius algebra $\left({ }^{3}\right)$.

(3) Tachikawa [15] has defined an algebra $A$ to be of cyclic-cocyclic type if for every indecomposable left $A$-module $M$, either $M / N M$ or $S(M)$ is simple. We shall prove that if $A$ is an algebra of cyclic-cocyclic type over an algebraically closed field $K$, such that $N^{2}=0$, then $A$ satisfies the hypothesis of our main theorem. Because the property of being an algebra of cyclic-cocyclic type, as well as the hypothesis of our main theorem, are satisfied by $A$ whenever they are satisfied by an algebra $A^{\prime}$ whose category of left modules is isomorphic to the category of left $A$-modules (see [12]), we can assume that $A$ is a basic algebra. Then the simple $A$-modules all have dimension 1 over $K$. Let $M$ be an indecomposable left $A$-module. If $S(M)$ is simple, then clearly $S(M)$ is square-free. Now suppose $S(M)$ is not square-free. We shall prove that $A$ is of unbounded module type. By hypothesis, $M / N M$ is simple. Then there exists an element $m \in M$ such that $M=A m$. Moreover there is a unique primitive idempotent $e$ in $A$ such that $e m \notin N m$, and we may assume $m=e m$. Since $M$ is indecomposable, and $N^{2}=0, N \neq 0$, we have $N m=S(M)$. Since $S(M)$ is not squarefree, there is a primitive idempotent $f$ such that $[f N m: K] \geqq 2$. Therefore $[f N e: K] \geqq 2$, and by an argument of Jans [8, pp. 421, 422], $A$ is of unbounded module type. This contradicts the assumption that $A$ is of cycliccocyclic type, and hence $S(M)$ must be square-free for all indecomposable modules $M$.

Tachikawa's results [15] show that the following algebra $A$ is of cycliccocyclic type. It is easily checked that $N^{2}=0$, and that since $A$ has a principal indecomposable left module without a unique minimal submodule, $A$ is neither generalized uniserial nor quasi-Frobenius. The algebra consists of all matrices

where $K$ is a field.

$$
\left(\begin{array}{lll}
x & 0 & 0 \\
u & y & 0 \\
v & 0 & z
\end{array}\right), \quad x, y, z, u, v \in K
$$

(4) Let $K$ be an algebraically closed field of characteristic 5, and let $G=\operatorname{LF}(2,5)$ be the simple group of order 60 . Then the 5-Sylow subgroup of $G$ is cyclic, so that the group algebra $K G$ is of bounded module type. The simple $K G$-modules, and the Cartan matrix of $K G$, are given in the paper of Brauer and Nesbitt [1]. From their results and a theorem of Morita [11, Lemma 1, p. 180], it follows that $K G$ is not generalized uni-

(3) Before the present paper was written, Curtis had investigated quasi-Frobenius algebras of bounded module type in collaboration with $\mathrm{H}$. Bass and D. Zelinsky, and he thanks both of them for a number of interesting and helpful ideas and suggestions on the subject. 
serial, although of course $K G$ is a quasi-Frobenius algebra. From Tachikawa's main theorem [15], it can be shown that $K G$ is not of cyclic-cocylic type. On the other hand a rather involved direct argument shows that the socle of every indecomposable left $K G$-module is square-free.

\section{REFERENCES}

1. R. Brauer and C. Nesbitt, On the modular characters of groups, Ann. of Math. (2) 42 (1941), 556-590.

2. H. Cartan and S. Eilenberg, Homological algebra, Princeton Univ. Press, Princeton, N.J., 1956.

3. C. W. Curtis, On algebras of bounded representation type, Abstract 597-141, Notices Amer. Math. Soc. 10 (1963), 99.

4. C. W. Curtis and I. Reiner, Representation theory of finite groups and associative algebras, Interscience, New York, 1962.

5. B. Eckmann and A. Schopf, Über injective Moduln, Arch. Math. 4 (1953), 75-78.

6. S. Eilenberg, Homological dimension and syzygies, Ann. of Math. (2) 64 (1956), 328-336.

7. D. G. Higman, Indecomposable representations at characteristic p, Duke Math. J. 21 (1954), 377-381.

8. J. P. Jans, On the indecomposable representations of algebras, Ann. of Math. (2) 66 (1957), 418-429.

9. _ Rings and homology, Holt, New York, 1964.

10. Some generalizations of finite projective dimension, Illinois J. Math. 5 (1961), 334-343.

11. K. Morita, On group rings over a modular field which possess radicals expressible as principal ideals, Sci. Rep. Tokyo Bunrika Daigaku 4 (1951), 177-194.

12. Duality for modules and its application to the theory of rings with minimum condition, Sci. Rep. Tokyo Kyoiku Daigaku Sect. A 6 (1958), 83-142.

13. T. Nakayama, Note on uni-serial and generalized uni-serial rings, Proc. Imp. Acad. Tokyo 16 (1940), 285-289.

14. R. J. Nunke, Modules of extensions over Dedekind rings, Illinois J. Math. 3 (1959), 222-241.

15. H. Tachikawa, On algebras of which every indecomposable representation has an irreducible one as the top or the bottom Loewy constituent, Math. Z. 75 (1961), 215-227.

16. T. Yoshii, Note on algebras of bounded representation type, Proc. Japan Acad. 32 (1956), 441-445.

UNIVERSITY OF WISCONSIN, Madison, Wisconsin

UNIVERSITY OF WASHINGTON,

SeAtTle, WashingtoN 